\title{
Resonant modal conversion in a two-mode waveguide
}

\author{
Yann G. Boucher • Alberto Parini . \\ Patrice Féron.
}

Received: date / Accepted: date

\begin{abstract}
We characterize a system consisting of a two-mode waveguide coupled to a single-mode microring resonator possibly presenting a nonlinear response of Kerr type. By using the scattering parameter formalism extended to the multimode domain, we show that in the linear regime and for an ideally transparent medium, each resonance of the system can be exploited to perform complete even-to-odd (respectively, odd-to-even) modal conversion. Moreover, when the Kerr nonlinearity is effective, the microring enables a power-dependent modal switching mediated by phase bistability. Thanks to its mode-processing capabilities, this configuration is suitable to find application as a functional building-block in mode-division multiplexing (MDM) photonic integrated circuits.
\end{abstract}

Keywords Mode Division Multiplexing - Optical Networks on Chip $(\mathrm{ONoCs}) \cdot$ Silicon photonics $\cdot$ Scattering parameters $\cdot$ Nonlinear resonators · Bistability

\section{Introduction}

A deep research effort is currently carried out by academic and industrial laboratories toward the integration on a same technological substrate of both microelectronic and photonic devices. In fact, as energy issues affect progressively the scalability of high-performance multi-core microprocessor architectures, the implementation of optical links (Optical Network-on-Chip - ONoC)

Y. G. Boucher

FOTON Photonic Systems, CNRS UMR 6082, F-22305 Lannion, France and also ENIB, Technopôle Brest-Iroise CS 73862, F-29238 Brest Cedex, France

Tel.: +330296469218

Fax: +330296370199

E-mail: yann.boucher@enib.fr

A. Parini and P. Féron

FOTON Photonic Systems, CNRS UMR 6082, University of Rennes 1, ENSSAT, F-22305 Lannion, France 
between computational cores is foreseen as a possible way to reduce the overall microprocessor power consumption while increasing at the same time the transmission bandwidth and data throughput (see [9], [1], [16] and references therein). The large majority of $\mathrm{ONoCs}$ solutions proposed in the literature rely for their operation on wavelength-division Multiplexing (WDM). WDM channels are easily routed, switched and multiplexed by means of functional devices, such as microring resonators [12], [17], [10] or Mach-Zehnder interferometers [23], [22], which are relatively easy to integrate on silicon photonic chips. The on-chip exploitation of WDM-based communication strategies implies the availability of silicon-compatible laser sources, eventually multi-wavelength. Although some recent remarkable fabrications have confirmed the technological feasibility of silicon photonic transceivers including III-V bonded laser sources [27], the integration of active cavities on silicon remains a challenging task, with the existing technological solutions providing contrasting performance in terms of available optical power, electric-to-optical power conversion efficiency and footprint. To reduce the number of sources or, in a complementary way, to optimize the use of the available ones, a possible solution relies on the exploitation of a further dimension inherent to optical communications, namely the modal order [11]. In mode-division multiplexing (MDM) techniques, communication channels are allocated on the same wavelength to the various propagation modes of a multi-mode waveguide. Therefore, used in conjunction with WDM, MDM can potentially increase the aggregate communication bandwidth of an optical link [7] and add a further degree of freedom for the design of on-chip integrated optical systems.

The implementation of a complete MDM on-chip interconnection system requires the definition of a full range of new functional building-blocks able to selectively process optical signals with respect to the spatial (modal) dimension. Such functional blocks may include add-drop mode multiplexers [5] [24], modeselective routers [18] and mode-exchangers. These last, in particular, play a key role as they can enable transparent reconfigurability of the transmission paths within the mode-multiplexed network. Different possible schemes enabling mode-exchange have been recently experimentally demonstrated in literature [19], [26]. In this work we specifically focus on a configuration consisting of a two-mode waveguide coupled to a single-mode resonator eventually presenting a nonlinear response of the Kerr type. Here, the mode-conversion functionality relies on the presence of the side-coupled resonator that, by breaking the invariance of the system, allows a power exchange between the even and odd modes of the two-mode waveguide, which would otherwise be orthogonal. Moreover, the possible presence of a nonlinear cubic (Kerr) response in the microring entails a dependence of the mode-conversion effect on the signal power through a bistable dynamic [4], [14], [6], [15]. A compact analytical expression for the transfer function of this configuration, providing also a full insight on its nonlinear behavior, is derived by observing that a multimode propagation can be interpreted as a coupling problem between single-mode fields. By exploiting this consideration, the scattering-parameter formalism, typically adopted for the dimensioning of single-mode devices and circuits, 


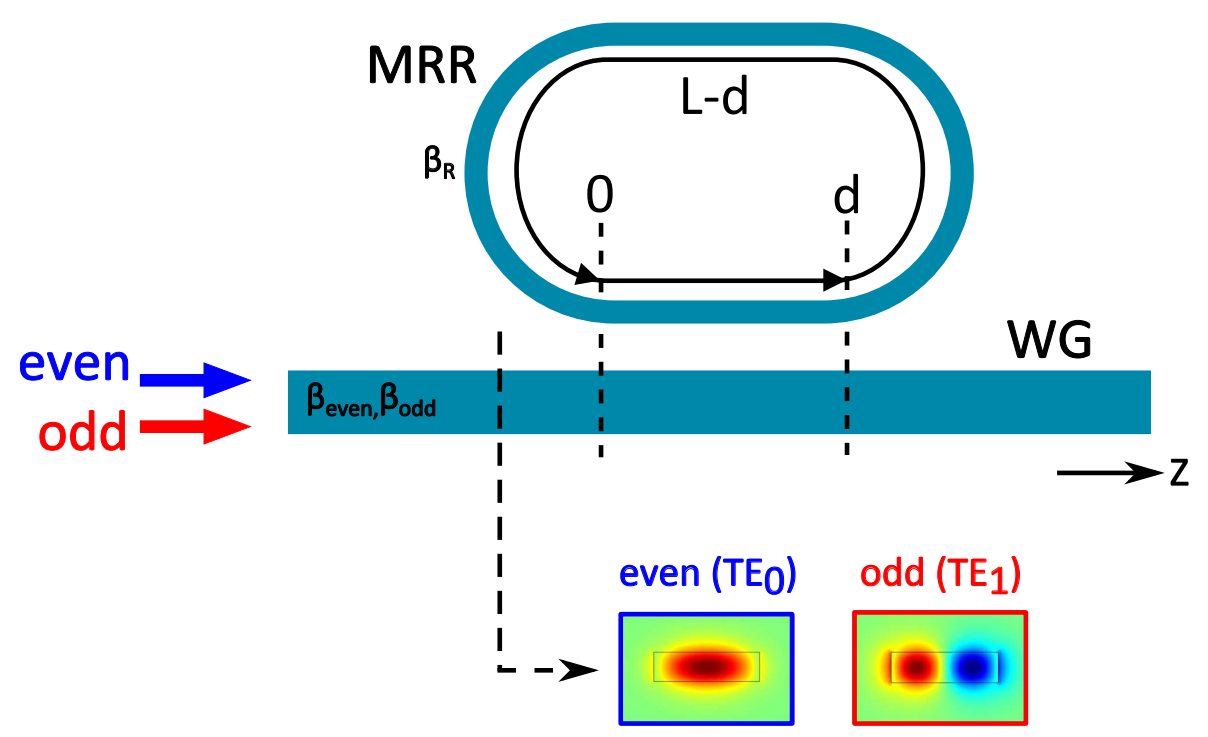

Fig. 1 Schematic representation of the system under investigation, consisting of a twomode waveguide $(W G)$ and a single-mode racetrack microring resonator $(M R R)$ of total perimeter $L$, which are evanescent coupled over a distance $d$.

can be extended to also model multi-mode configurations. The article is organized as follows: In Section (2) the system configuration is presented, together with the definition of the relevant governing parameters. In Section (3), the formal equivalence between a two-mode waveguide and a system of two coupled single-mode waveguide is outlined. In Section (4) the interaction zone between the two-mode waveguide and the microring resonator is described in terms of a ternary single-mode directional coupler. In Section (5) the modal transfer function of the entire system is established in a compact matrix form. Finally, in Section (6), the investigation is extended to the nonlinear domain by introducing a Kerr response in the microring resonator. Conclusions and perspectives are drawn at the end.

\section{System layout and governing parameters}

The system under investigation consists of a two-mode waveguide (WG), coupled to a single-mode racetrack microring resonator (MRR) of total perimeter $L$, as depicted in Fig. 1. Evanescent coupling between the two-mode waveguide and the single-mode microring takes place along an interaction zone of total length $d$. For the sake of clarity, we limit the discussion to a single eigenstate of polarization, either TE or TM, so that each mode can be represented by an associated scalar value. The time dependence is taken as $\exp (+i \omega t)$ and $z$ is the direction of propagation in the straight waveguide. The two-mode waveguide is dimensioned to guide a fundamental mode with even symmetry and a first-order mode with odd symmetry, both characterized by their respective 
propagation constants $\beta_{\text {even }}$ and $\beta_{\text {odd }}$, with $\beta_{\text {even }}>\beta_{\text {odd }}$. We consider here a device designed to perform on-chip optical signal processing on a CMOS compatible platform, therefore we identify the even and odd modes as the $T E_{0}$ and the $T E_{1}$ modes of a multi-mode silicon on insulator (SOI) waveguide. In order to derive for this system an analytical input-output relation under the form of a scattering (Jones-like) matrix, we exploit the equivalence that can be established between a two-mode waveguide and a set of two coupled single-mode waveguides [2], as will be detailed in the next section.

\section{Equivalence between a two-mode waveguide and a symmetric single-mode coupler}

In the framework of coupled-mode theory (CMT) [25], [20], the periodic power exchange that takes place in a symmetric coupler between two single-mode waveguides separated by a distance $g$, such as the one schematized in Fig. 2(A), can be interpreted as a pattern resulting from the simultaneous propagation of the even and odd super-modes of the structure. The common propagation constant $\beta$ of the two single-mode waveguides, and their mutual coupling strength $\chi$, can be related to the propagation constant $\beta_{\text {even }}$, and $\beta_{\text {odd }}$ of the two super-modes by the following exact relations:

$$
\begin{aligned}
& \beta=\frac{\beta_{\text {even }}+\beta_{\text {odd }}}{2}, \\
& \chi=\frac{\beta_{\text {even }}-\beta_{\text {odd }}}{2} .
\end{aligned}
$$

In the limiting case of a vanishing gap $(g=0)$, the even and odd super-modes of the coupler progressively converge toward the fundamental (even-symmetry) and first order (odd-symmetry) modes of a two-mode waveguide, as illustrated in Fig. 2(B). This allows us to say that a two-mode waveguide can also be thought of as a system of two tightly coupled single-mode waveguides, in which the gap distance has collapsed to zero [2]. Moreover, from the knowledge of the propagation constants of the even and odd modes (the $T E_{0}$ and $T E_{1}$ ones in the SOI case), and by exploiting Eqs. (1) and (2), it is straightforward to establish an equivalent description for the two-mode waveguide involving only two single-mode structures, as will be detailed in the subsequent sections.

\section{Scattering matrix model of the coupling section}

By exploiting the formal equivalence outlined in the previous section, the coupling zone between ring and two-mode waveguide of the initial configuration, as depicted in Fig. 3(A), can be substituted with an asymmetric ternary coupler between three single-mode waveguides, as represented in Fig. 3(B). In this equivalent framework, $W G_{1}$ is the waveguide belonging to the microring 

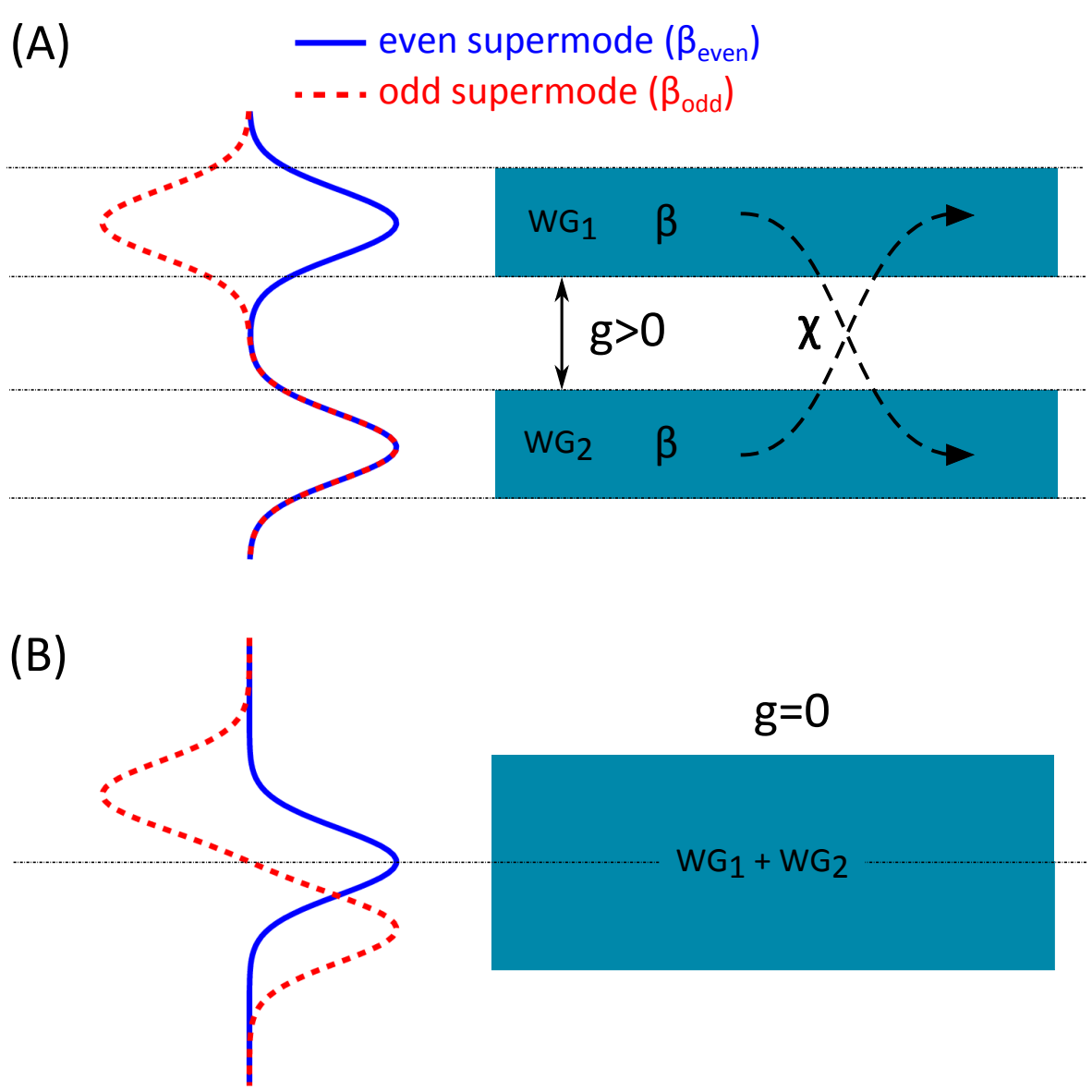

Fig. 2 The even (blue) and odd (red) supermodes of a symmetric coupler between two single-mode waveguides (A) characterized by the same propagation constant $\beta$ and exchanging power with a coupling coefficient $\chi$, converge towards the even and odd modes of a two-mode waveguide (B) as the gap $g$ progressively vanishes. Conversely, any two mode waveguide can therefore be interpreted as a symmetric single-mode coupler with $g=0$.

resonator, while $W G_{2}$ and $W G_{3}$ are the two new single-mode waveguides resulting from the decomposition of the original two-mode section.

According to Eqs. (1) and (2), the common propagation constant of both $W G_{2}$ and $W G_{3}$ is $\beta=\left(\beta_{\text {even }}+\beta_{\text {odd }}\right) / 2$ and their mutual coupling coefficient is $\chi_{b}=\left(\beta_{\text {even }}-\beta_{\text {odd }}\right) / 2, \beta_{\text {even }}$ and $\beta_{\text {odd }}$ being the propagation constants of the even and odd modes of the initial two-mode waveguide, respectively. Moreover, for the sake of simplicity, we assume perfect phase matching between the microring and the decomposed single-mode waveguides, so $W G_{1}$ shares the same propagation constant $\beta$. The interaction between the microring resonator $\left(W G_{1}\right)$ and $W G_{2}$ is accounted for by a second coupling coefficient $\chi_{a}$. 
We further assume that coupling takes place only between neighboring waveguides (i.e. no direct coupling between $W G_{1}$ and $\left.W G_{3}\right)^{1}$.

We define $F_{1}, F_{2}$ and $F_{3}$ as the waves propagating in the three waveguides, with:

$$
\begin{aligned}
& F_{1}(0)=a_{1} \\
& F_{2}(0)=a_{2} \\
& F_{3}(0)=a_{3},
\end{aligned}
$$

and

$$
\begin{aligned}
& F_{1}(d)=b_{4} \\
& F_{2}(d)=b_{5} \\
& F_{3}(d)=b_{6},
\end{aligned}
$$

so that $a_{n}$ (respectively $b_{n}$ ) represents the complex amplitude of the wave entering into (respectively coming out of) the $n$-th port of the ternary coupler of length $d$. The propagation of $F_{n}$ waves within the ternary coupler (assuming the $W G_{1}$ ring waveguide to be open) obeys the following matrix equation:

$$
i \frac{\partial}{\partial z}\left(\begin{array}{l}
F_{1} \\
F_{2} \\
F_{3}
\end{array}\right)=\left(\begin{array}{ccc}
\beta & \chi_{a} & 0 \\
\chi_{a} & \beta & \chi_{b} \\
0 & \chi_{b} & \beta
\end{array}\right)\left(\begin{array}{l}
F_{1} \\
F_{2} \\
F_{3}
\end{array}\right) .
$$

It is more convenient to work in the framework of the slowly-varying envelopes $A_{n}$, with $F_{n}=A_{n} \exp (-i \beta z)$ and to define two new parameters $\chi=\sqrt{\chi_{a}^{2}+\chi_{b}^{2}}$ and $\alpha$, such as:

$$
\begin{aligned}
& \chi_{a}=\chi \sin (\alpha) \\
& \chi_{b}=\chi \cos (\alpha) .
\end{aligned}
$$

Since the coupling strength between the microring and the two-mode waveguide is weaker than the coupling strength between the two propagating modes inside the two-mode waveguide (in the even-odd supermodes framework), we can assume $\chi_{a} \leq \chi_{b}$ with $\alpha \in[0, \pi / 4]$. Eq. (5) can be rewritten under the following form:

$$
i \frac{\partial}{\partial z}\left(\begin{array}{l}
A_{1} \\
A_{2} \\
A_{3}
\end{array}\right)=\chi\left(\begin{array}{ccc}
0 & \sin \alpha & 0 \\
\sin \alpha & 0 & \cos \alpha \\
0 & \cos \alpha & 0
\end{array}\right)\left(\begin{array}{l}
A_{1} \\
A_{2} \\
A_{3}
\end{array}\right)
$$

Taking Eq. (7) as a starting point, we follow a standard diagonalization procedure of the operator to derive its eigenvalues and eigenvectors. After some

\footnotetext{
1 By means of simple analytical evaluations made on slab waveguides, one can quickly verify the validity of this assumption, as long as $W G_{1}$ remains reasonably far from the system made by $W G_{2}+W G_{3}$. In fact, given the transverse power distribution of the modes in this configuration, the overlap integral between $W G_{1}$ and $W G_{3}$ (which is roughly proportional to the coupling constant) appears to be vanishingly smaller than the one between $W G_{1}$ and $W G_{2}$.
} 

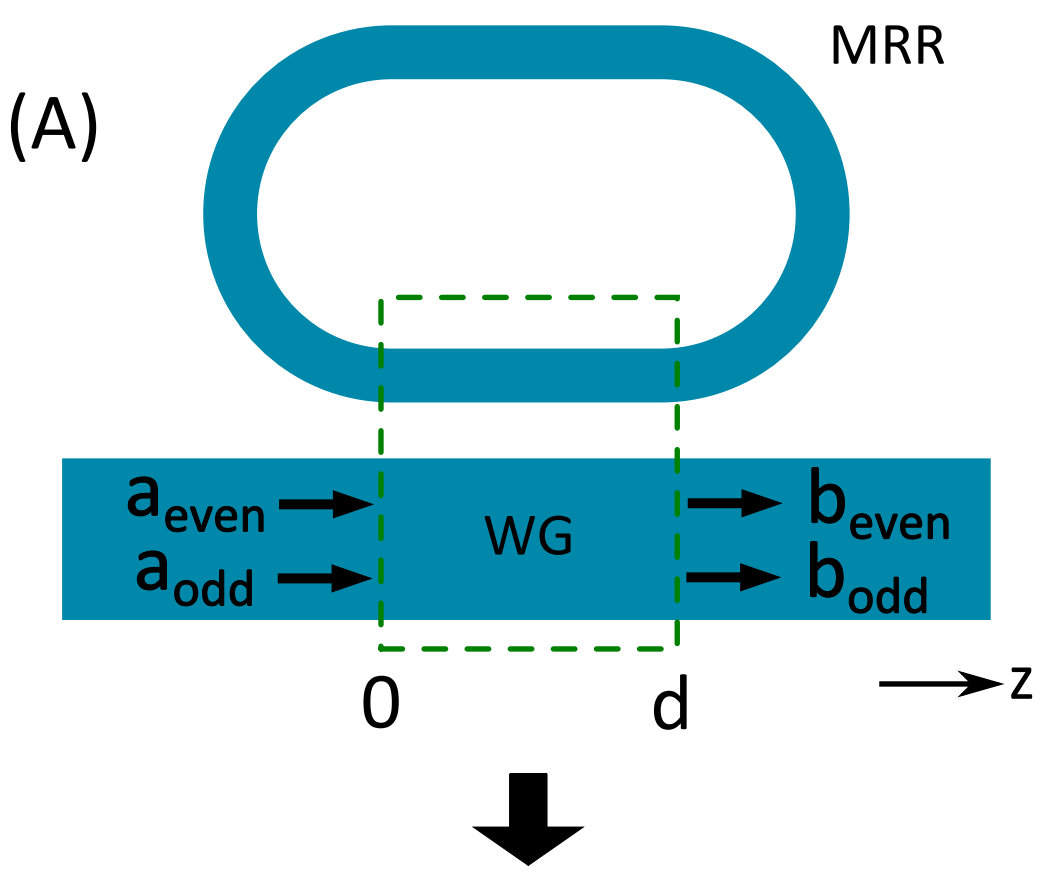

(B)
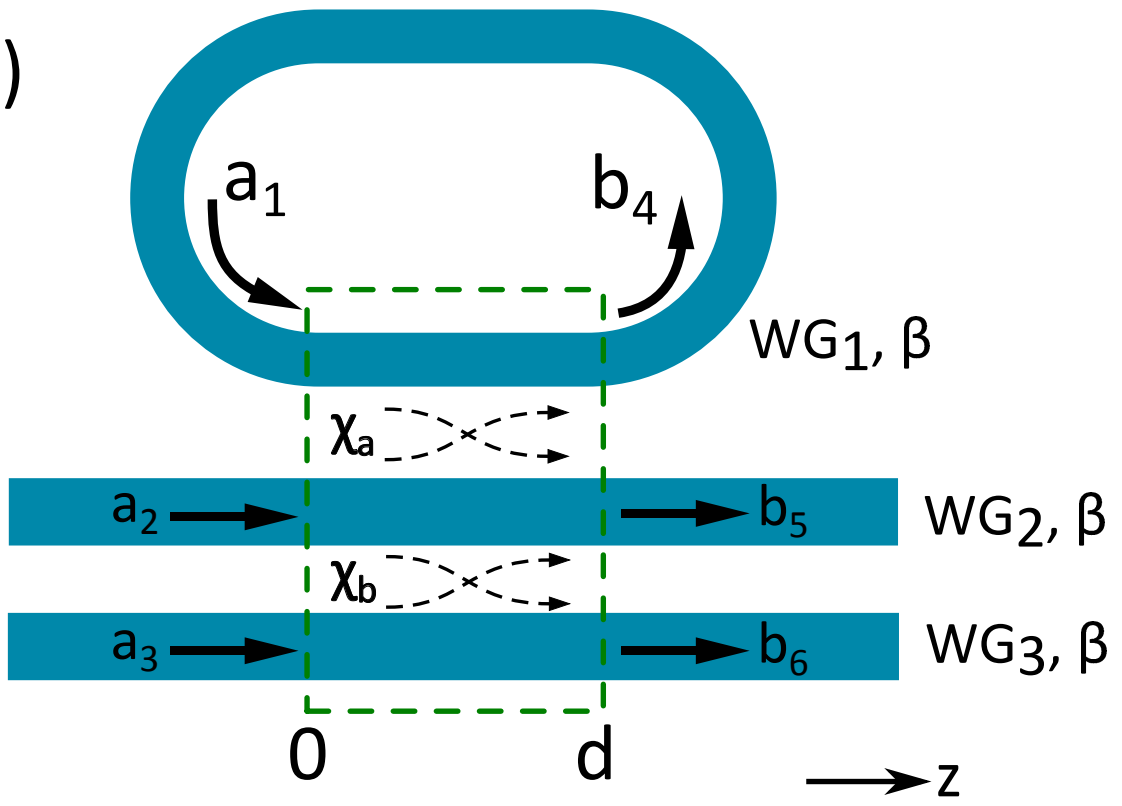

Fig. 3 The initial configuration of the system (A) is described by the even and odd mode amplitudes at the input $\left(a_{\text {even }}, a_{\text {odd }}\right)$ and output $\left(b_{\text {even }}, b_{\text {odd }}\right)$ ports. After substitution of the two-mode waveguide with an equivalent coupler between two single-mode ones, the interaction zone highlighted by the green-dotted rectangle is modelled as a ternary directional coupler between three single-mode waveguides $\left(W G_{1}, W G_{2}\right.$ and $\left.W G_{3}\right)$ which share the same propagation constant $\beta$. Coupling is supposed to take place only between neighboring waveguides with strength $\chi_{a}$ between $W G_{1}$ and $W G_{2}$ and strength $\chi_{b}$ between $W G_{2}$ and $W G_{3}$. 
calculations, we obtain eventually the following scattering matrix representation, which relates the complex field amplitudes $a_{n}$ and $b_{n}$ of the ternary coupler on the initial $(z=0)$ and final $(z=d)$ sections, respectively:

$$
\left(\begin{array}{l}
b_{4} \\
b_{5} \\
b_{6}
\end{array}\right)=\mathrm{e}^{-i \beta d}\left(\begin{array}{ccc}
\rho & -i \kappa & X \\
-i \kappa & \tau & -i \kappa^{\prime} \\
X & -i \kappa^{\prime} & \rho^{\prime}
\end{array}\right)\left(\begin{array}{l}
a_{1} \\
a_{2} \\
a_{3}
\end{array}\right),
$$

where we define the following parameters:

$$
\begin{aligned}
\theta & =\chi d \\
\rho & =\sin ^{2} \alpha \cos \theta+\cos ^{2} \alpha \\
\kappa & =\sin \alpha \sin \theta \\
X & =\sin \alpha \cos \alpha(\cos \theta-1) \\
\tau & =\cos \theta \\
\rho^{\prime} & =\cos ^{2} \alpha \cos \theta+\sin ^{2} \alpha \\
\kappa^{\prime} & =\cos \alpha \sin \theta
\end{aligned}
$$

Beside this, it can also be checked that $1-\rho=\rho^{\prime}-\tau=\sin ^{2} \alpha(1-\cos \theta)$, which will be useful in the calculations of Section 5 .

It must be observed that an equivalent matrix expression for the coupler could have been derived starting from a different basis set, namely the one explicitly dealing with even and odd mode amplitudes in the two-mode waveguide. However, our reference system made of single mode waveguides makes the ternary coupler simpler to model, as detailed in Appendix (A) .

\section{Modal transfer function of the system}

By operating a substitution of the two-mode waveguide with its equivalent single-mode representation, we have derived the scattering matrix (8) which describes the mode-amplitude evolution within the ternary coupler, assuming the $W G_{1}$ waveguide to be open. A matrix expression accounting also for the presence of the microring ( $W G_{1}$ closed on itself) can be derived by observing that:

$$
a_{1}=b_{4} \gamma \mathrm{e}^{-i \beta(L-d)},
$$

where $\gamma$ is the propagation loss factor over the distance $(L-d)$.

By introducing Eq. (10) in Eq. (8), we obtain the following $(2 \times 2)$ matrix expression:

$$
\left(\begin{array}{l}
b_{5} \\
b_{6}
\end{array}\right)=\left(\begin{array}{cc}
A & -i B \\
-i B & C
\end{array}\right)\left(\begin{array}{l}
a_{2} \\
a_{3}
\end{array}\right),
$$


which links directly the $\left(a_{1}, a_{2}\right)$ and $\left(b_{5}, b_{6}\right)$ mode amplitudes. The elements in 11 are:

$$
\begin{aligned}
& A=\mathrm{e}^{-i \beta d}\left(\tau-\kappa^{2} \frac{\gamma \mathrm{e}^{-i \beta L}}{1-\rho \gamma \mathrm{e}^{-i \beta L}}\right), \\
& B=\mathrm{e}^{-i \beta d}\left(\kappa^{\prime}+\kappa X \frac{\gamma \mathrm{e}^{-i \beta L}}{1-\rho \gamma \mathrm{e}^{-i \beta L}}\right), \\
& C=\mathrm{e}^{-i \beta d}\left(\rho^{\prime}+X^{2} \frac{\gamma \mathrm{e}^{-i \beta L}}{1-\rho \gamma \mathrm{e}^{-i \beta L}}\right) .
\end{aligned}
$$

For the sake of clarity, we stress again that Eq. (11) relates the field amplitude on the $z=0$ and $z=d$ sections of the ternary coupler, when $W G_{1}$ is closed on itself (closed loop) in the framework where the two-mode waveguide is interpreted as a system of two tightly coupled single-mode waveguides. In order to switch back to the initial even-odd mode amplitude representation, the following rotation matrices can be straightforwardly applied to Eq. (11):

$$
\begin{aligned}
\left(\begin{array}{c}
b_{\text {even }} \\
b_{\text {odd }}
\end{array}\right) & =\frac{\sqrt{2}}{2}\left(\begin{array}{cc}
1 & 1 \\
-1 & 1
\end{array}\right)\left(\begin{array}{l}
b_{5} \\
b_{6}
\end{array}\right), \\
\left(\begin{array}{c}
a_{2} \\
a_{3}
\end{array}\right) & =\frac{\sqrt{2}}{2}\left(\begin{array}{cc}
1 & -1 \\
1 & 1
\end{array}\right)\left(\begin{array}{c}
a_{\text {even }} \\
a_{\text {odd }}
\end{array}\right),
\end{aligned}
$$

leading to the matrix expression:

$$
\left(\begin{array}{c}
b_{\text {even }} \\
b_{\text {odd }}
\end{array}\right)=[\mathrm{J}]\left(\begin{array}{c}
a_{\text {even }} \\
a_{\text {odd }}
\end{array}\right)=\frac{1}{2}\left(\begin{array}{cc}
A+C-2 i B & -A+C \\
-A+C & A+C+2 i B
\end{array}\right)\left(\begin{array}{c}
a_{\text {even }} \\
a_{\text {odd }}
\end{array}\right),
$$

which relates the even/odd mode amplitudes between the $z=0$ and $z=d$ sections of the device. The explicit form for the elements of $[J]$ reads as:

$$
\begin{aligned}
& J_{11}=\frac{\mathrm{e}^{-i \beta d}}{2}\left(\rho^{\prime}+\tau-2 i \kappa^{\prime}+\frac{\gamma \mathrm{e}^{-i \beta L}}{D}(X-i \kappa)^{2}\right), \\
& J_{12}=J_{21}=\frac{\mathrm{e}^{-i \beta d}}{2}\left(\rho^{\prime}-\tau+\frac{\gamma \mathrm{e}^{-i \beta L}}{D}\left(X^{2}+\kappa^{2}\right)\right), \\
& J_{22}=\frac{\mathrm{e}^{-i \beta d}}{2}\left(\rho^{\prime}+\tau+2 i \kappa^{\prime}+\frac{\gamma \mathrm{e}^{-i \beta L}}{D}(X+i \kappa)^{2}\right),
\end{aligned}
$$

where $D=1-\rho \gamma \mathrm{e}^{-i \beta L}$ is a common denominator factor characterizing the microring. As the elements of the matrix $J$ depend on the spectral parameter $\beta$, the degree of mode mixing produced by the system depends intrinsically on the working wavelength. In the specific case of a transparent microring $(\gamma=1)$ and for wavelengths corresponding to one of the resonances of the microring $[\exp (-i \beta L)=1]$, since $D=1-\rho$ the elements of $J$ reduce to:

$$
\begin{aligned}
& J_{11}=J_{22}=0, \\
& J_{12}=J_{21}=\mathrm{e}^{-i \beta d} .
\end{aligned}
$$


The matrix $J$ has therefore an anti-diagonal structure, thus enabling a complete even-odd (odd-even) cross-coupling between the modes as they transit through the system. More explicitly, an even (odd) mode incident on the input port ( $\operatorname{section} z=0$ ) is transmitted to the output port (section $z=d$ ) as an odd (even) one. Conversely, on the anti-resonance wavelengths, for which $[\exp (-i \beta L)=-1]$, the vanishing terms of the matrix $J$ are the anti-diagonal ones $\left(J_{12}=J_{21}=0\right)$ and any incident modal state is transmitted unchanged as a result of the diagonal shape of $J$. In Fig. (4) are presented the evolutions of the $b_{\text {even }}$ (thick blue line) and $b_{\text {odd }}$ (thin red line) output mode amplitudes calculated directly through Eq. (14) as a function of the parameter $\beta L$ in the range $[-3 \pi, 3 \pi]$ in the case of a pure even mode excitation on the input port $\left(a_{\text {even }}=1, a_{\text {odd }}=0\right)$. As expected, on each of the microring resonances [where $(\beta L=2 q \pi)$, with $q$ integer], the even mode power on the input port is completely converted to the odd mode on the output port $\left(b_{o d d}=1\right)$ while on the anti-resonances [where $(\beta L=(2 q+1) \pi)$ ] no conversion takes place. Moreover, between resonance and anti-resonance conditions, an intermediate degree of mode mixing is achievable. We also observe that, following the assumption of a transparent media, the total power carried by even and odd modes is conserved through the passage within the system $\left(\left|a_{\text {even }}\right|^{2}+\left|a_{\text {odd }}\right|^{2}=\left|b_{\text {even }}\right|^{2}+\left|b_{\text {odd }}\right|^{2}=\right.$ const $)$, the only change concerns the modal repartition.

In view of the forthcoming nonlinear modelling, it is useful to derive a closed expression for the field amplitude $b_{4}$ inside the resonator as a function of the mode amplitudes $a_{2}$ and $a_{3}$ :

$$
b_{4}=\mathrm{e}^{-i \beta d}\left(\frac{-i \kappa a_{2}+X a_{3}}{1-\rho \gamma \mathrm{e}^{-i \beta L}}\right),
$$

or, alternatively, as a function of the even/odd input mode amplitudes:

$$
b_{4}=a_{\text {even }} \frac{\sqrt{2}}{2} \mathrm{e}^{-i \beta d}\left(\frac{X-i \kappa}{1-\rho \gamma \mathrm{e}^{-i \beta L}}\right)+a_{o d d} \frac{\sqrt{2}}{2} \mathrm{e}^{-i \beta d}\left(\frac{X+i \kappa}{1-\rho \gamma \mathrm{e}^{-i \beta L}}\right)
$$

The power carried by the system depends on the possible coherence between the even and odd modes. If these are mutually incoherent, then the internal power is reduced to the mere sum of the even and odd contributions. Moreover, as a result of the phase quadrature between factors $X$ and $i \kappa$, both even and odd modes are equally coupled into the ring.

\section{Nonlinear regime}

6.1 Phase bistability and mode conversion

In the previous sections of this work, we have derived a matrix expression relating the amplitudes of the even/odd modes at the input-output ports of the device and we have shown that, under particular conditions, this system can 


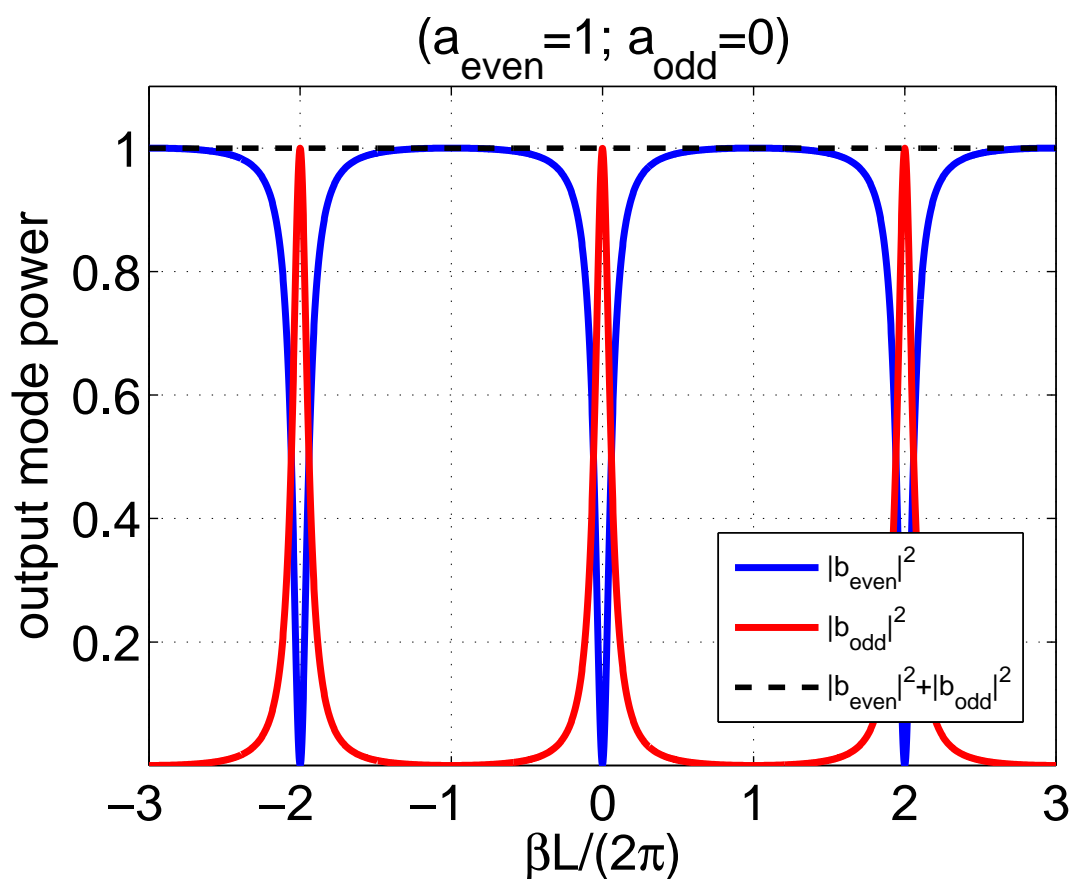

Fig. 4 Even (blue thick solid line) and odd (red thin solid line) output mode evolution versus $\beta L$, for system parameters $\chi_{a}=\chi_{b}=0.6, d=1, L=20, a_{\text {even }}=1$ and $a_{\text {odd }}=0$ (i.e. pure even excitation on the input port). The dashed black line represents the total (conserved) modal power.

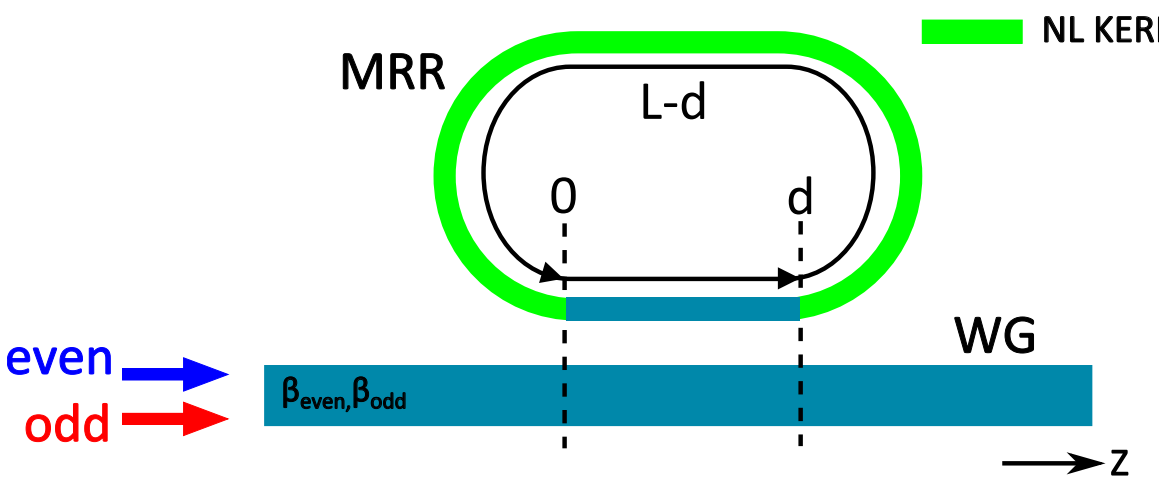

Fig. 5 Investigated configuration in the nonlinear Kerr domain; the coupling zone of length $d$ is supposed to be linear.

act as an ideal mode converter. We now extend our discussion by considering a microring resonator presenting a nonlinear response of the Kerr type, as schematically shown in Fig. 5. We assume (i) the nonlinear zone perfectly matched at both ends, so that the whole system remains unidirectional and (ii) that the coupling zone of length $d$ is still linear. For the sake of clarity, we 
consider a strictly even input mode on the left port of the device $\left(a_{\text {odd }}=0\right)$, and a purely loss-less microring $(\gamma=1)$.

The Kerr medium introduces an additional nonlinear phase shift which is proportional to the field power $\left|b_{4}\right|^{2}$ circulating in the microring. It is useful to introduce the reduced (dimensionless) quantity:

$$
Y_{\text {int }}=\frac{\left|b_{4}\right|^{2}}{P_{\text {NORM }}},
$$

normalized with respect to a term $P_{N O R M}$ chosen in such a way that the total internal phase shift $\varphi$ in the microring (comprehensive of both linear and nonlinear contributions) could be expressed as:

$$
\varphi=\varphi_{\text {lin }}+2 \pi Y_{\text {int }} .
$$

By defining $Y_{e}=\left|a_{e v e n}\right|^{2} / P_{N O R M}$ as the reduced input power for the (even) excitation, the power within the ring $Y_{i n t}$ reads as:

$$
Y_{\text {int }}=Y_{e} K_{0} A(\varphi) \text {, }
$$

where $K_{0}$ is a coupling factor that depends only on the coupler characteristics:

$$
K_{0}=\frac{|X-i \kappa|^{2}}{2(1-\rho)^{2}}=\frac{1}{2}\left(\frac{1+\rho}{1-\rho}\right),
$$

and $A(\varphi)$ is a normalized comb-like Airy function

$$
A(\varphi)=\frac{1}{1+m \sin ^{2}(\varphi / 2)},
$$

whose spectral selectivity depends on the parameter $m$

$$
m=\frac{4 \rho}{(1-\rho)^{2}} .
$$

We would like to emphasize that, for a given reduced input power $Y_{e}$, neither the microring internal power $Y_{\text {int }}$ nor the total phase shift $\varphi$ are known quantities, this being the nonlinear nature of the problem. To determine $Y_{\text {int }}$ and $\varphi$ in a self-consistent way, we need to suitably rewrite the expressions (20) and (21) in order to put in evidence their simultaneous dependence on the unknown parameter $\varphi$ :

$$
\begin{aligned}
\frac{Y_{i n t}}{Y_{e}} & =K_{0} A(\varphi), \\
\frac{Y_{i n t}}{Y_{e}} & =\frac{\varphi-\varphi_{\text {lin }}}{2 \pi Y_{e}} .
\end{aligned}
$$

The right-hand term of Eq. (25a) has the form of a comb-like Airy function, while the right-hand term of Eq. (25b) describes a family of straight lines whose slope is inversely proportional to $Y_{e}$. 


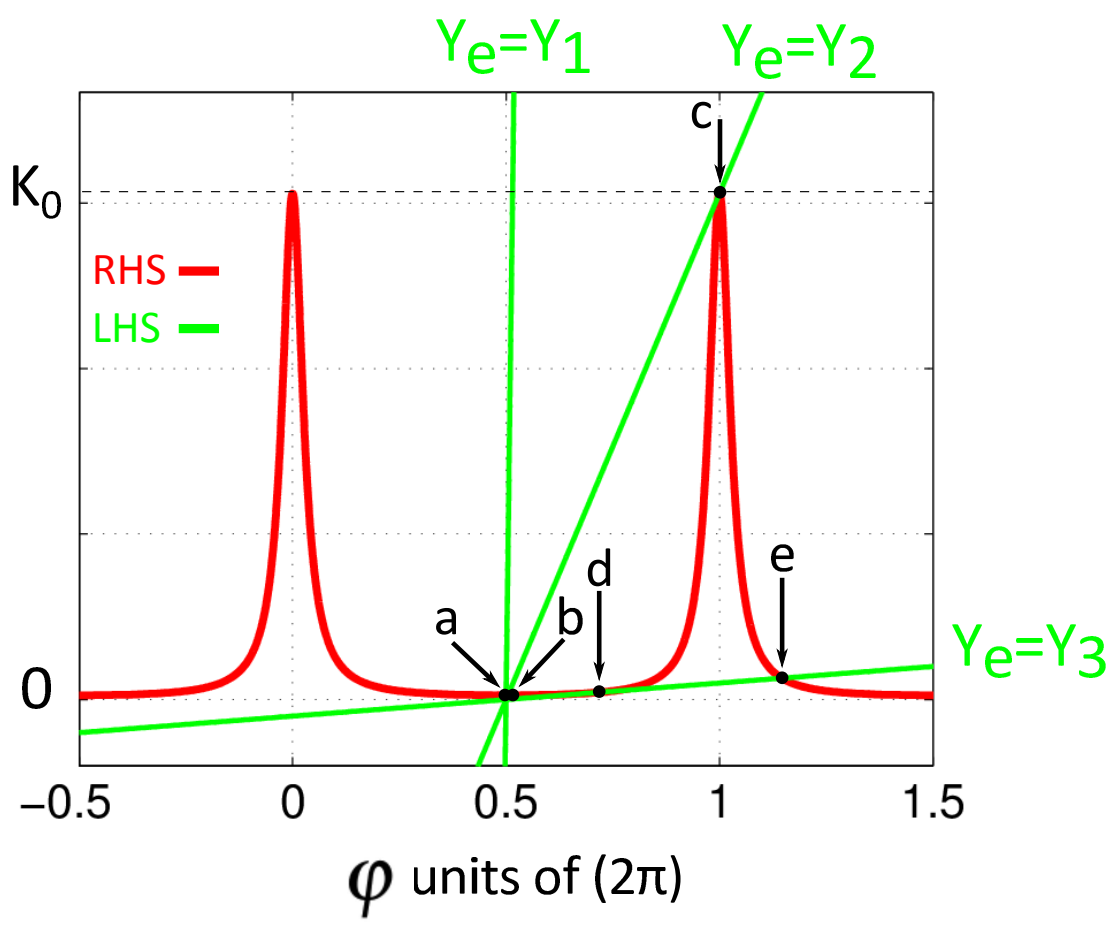

Fig. 6 RHS (red solid line) and LHS (green solid line) of Eq. (26) traced against the nonlinear phase shift $\varphi$. Here the parameters of the system are: $\chi_{a}=\chi_{b}=0.6, d=1, L=20$, $a_{\text {even }}=1$ and $a_{\text {odd }}=0$.

By equating (25a) and (25b) we get the following expression in the unknown total phase shift $\varphi$ :

$$
\frac{K_{0}}{1+m \sin ^{2}(\varphi / 2)}=\frac{\varphi-\varphi_{\text {lin }}}{2 \pi Y_{e}},
$$

whose solution(s) as a function of $Y_{e}$ can be found either graphically [3] or numerically. Moreover, if the microring resonances are sharp enough to be assimilated to Lorentzian functions, these solutions can be also derived analytically as the roots of a third order algebraic equation (see Appendix B). The system behavior as a function of the reduced input power $Y_{e}$ can be assessed by referring to Fig. 6 , where the left-hand side (LHS) and the right-hand side (RHS) of Eq. (26) are plotted separately against the unknown total phase shift $\varphi$. Intersection points between RHS and LHS represent the graphical solutions of Eq. (26) and their projections of on the x-axis give directly the total phase shift values associated to the different input powers $Y_{e}$. For lower input power $\left(Y_{e}=Y_{1}\right)$, the RHS straight line intersects the LHS curve only in $(a)$ and its projection on the $\mathrm{x}$-axis identifies the total phase shift of the microring in the quasi linear regime $\left(\varphi=\varphi_{\text {lin }}\right)$. When the input power $Y_{e}$ is increased $\left(Y_{e}=Y_{2}\right)$, the slope of the RHS line decreases progressively until intersecting the LHS curve simultaneously in $(b)$ and $(c)$. In this conditions Eq. (26) admits 


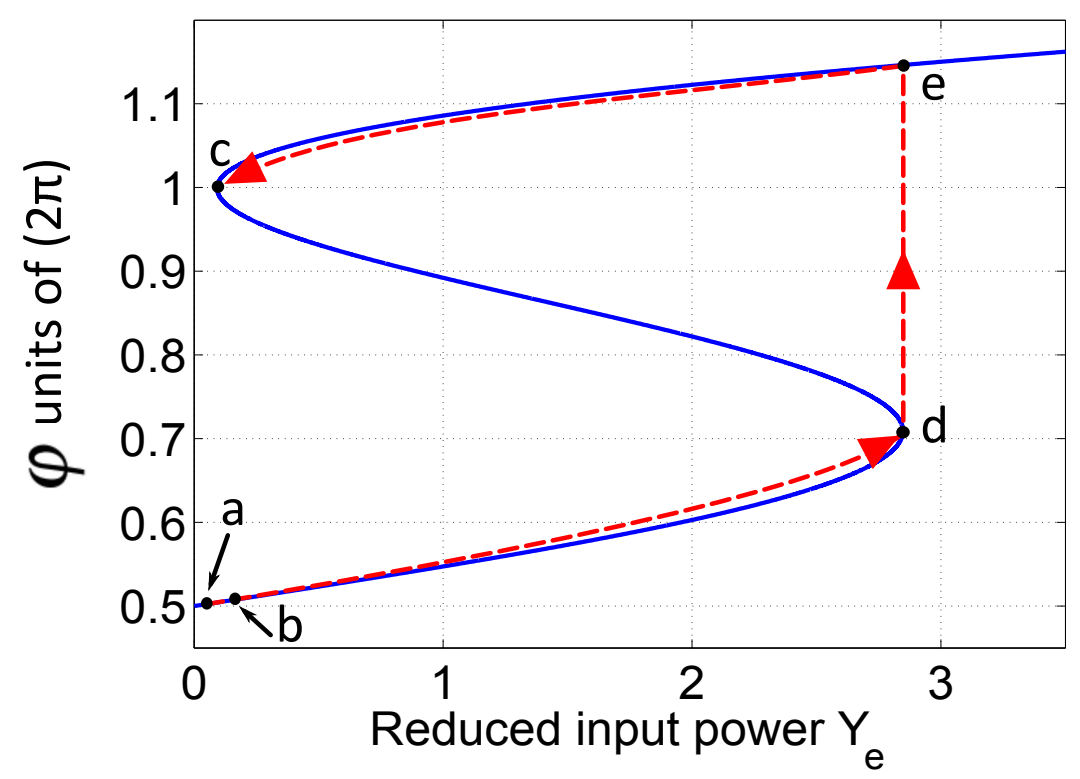

Fig. 7 (Blue solid line) Evolution of the microring total phase shift $\varphi$ against the (purely even) reduced input power $Y_{e}$. The points of the curve are obtained by numerically solving Eq. (26). When the reduced input power $Y_{e}$ follows the path highlighted by the red dashed arrows, the system evolves from the working point $(a)$ (microring off-resonance, no mode conversion) to the working point $(c)$ (microring on resonance, complete mode conversion). The system parameters are the same as in Fig. 6

two possible solutions which entail a bistable behavior. A further input power increase $\left(Y_{e}=Y_{3}\right)$ makes the system evolve toward further solution points $(d, e)$ thus tracing a complete hysteresis loop. It is now important to observe that the intersection point $(c)$ lies exactly on the peak of the RHS function, and that the total phase shift associated to this solution is $\varphi=2 \pi$. In this specific working point the microring is in a resonance condition and the matrix $J$ has, consequently, a diagonal structure which enables a complete mode conversion. A possible functionalization of such a system is clearly a nonlinear mode switch (or modal commutator). In fact, if the linear working point is initially set to an anti-resonance of the microring $\left(\varphi=\varphi_{\text {lin }}=\pi\right)$, as the input power $Y_{e}$ of the mode is increased, the system is led from an initial condition where no mode conversion takes place ( $J$ in diagonal form), to a condition of complete mode conversion ( $J$ in anti-diagonal form). More explicitly, an even (odd) mode tuned to an anti-resonance in the linear limit, would switch to an odd (even) mode as the system is progressively driven through its hysteresis cycle. The bistable nature of the system is clearly revealed in Fig. 7, where the values of the total phase shift $\varphi$ (blue solid line) are traced for increasing values of the reduced (even-mode) input power $Y_{e}$. On the same curve, also the solution points $(a),(b),(c),(d)$ and $(e)$ previously discussed in Fig. 6 are reported. 
As one can note, by following with the input power $Y_{e}$ the path highlighted by the red dashed line, the system can be led from the point $(a)$, corresponding to a condition of mode decoupling (no mode conversion), to the point (c) where the mode conversion is complete, thus confirming the potential applicability of this configuration as a power-controlled mode switcher/converter.

\subsection{Relation between Q-factor and mode-conversion power level}

For a practical implementation of the device, it is useful to assess the dependence between the microring quality factor $\mathrm{Q}$, which finally quantifies the maximum possible bandwidth of the signal to be processed, and the input power level $Y_{e}$ of the point $(c)$ where mode conversion takes place.

By observing Fig. (6), one can note that the (c) point has Cartesian coordinates $(x, y)=\left(2 q \pi, K_{0}\right)$ with $q$ integer, and that on each of the microring resonances (for which $\varphi=2 q \pi$ ), the value of the corresponding reduced input power can be directly derived by simple inversion of Eq. (26):

$$
Y_{e}=\frac{2 q \pi-\varphi_{\text {lin }}}{K_{0}}=\left(4 q \pi-2 \varphi_{\text {lin }}\right)\left(\frac{1-\rho}{1+\rho}\right),
$$

in which the explicit dependence from the $\rho$ parameter is obtained through Eq. (22).

As long as $m$ is high enough to have well defined Lorentzian lineshapes, also the quality factor $Q$ of the resonator is linked to the coupling coefficient $\rho$ through the following relation:

$$
Q=\frac{\varphi_{0}}{2}\left(\frac{\sqrt{\rho}}{1-\rho}\right)=q \pi\left(\frac{\sqrt{\rho}}{1-\rho}\right),
$$

where $\varphi_{0}=2 q \pi$ is the actual phase of the resonance peak chosen as working point.

It should now be remembered that $\rho$ represents the fraction of field which is reinjected in the ring at each loop (Eq. 8), therefore, an increase (decrease) of $\rho$ produces an increase (decrease) of the $K_{0}$ and $Q$ parameters via Eqs. $(22),(28)$ with a corresponding decrease (increase) through Eq. (27) of the $Y_{e}$ reduced point corresponding to the $(c)$ point, as graphically evidenced in Fig. (8). As the $\rho$ coefficient approaches progressively to unity, the quality factor $Q$ grows towards infinity with a consequent enhancement of the nonlinear effect at the expenses of a reduced available bandwidth for signal transmission. This dynamic is therefore typical of resonant systems encompassing third order (Kerr) effects, as already evidenced in literature at both analytical and experimental levels [13], [21], [8].

\section{Conclusions}

In summary, we have established the scattering matrix model of a two-mode waveguide coupled to a single-mode racetrack ring resonator, in both linear 


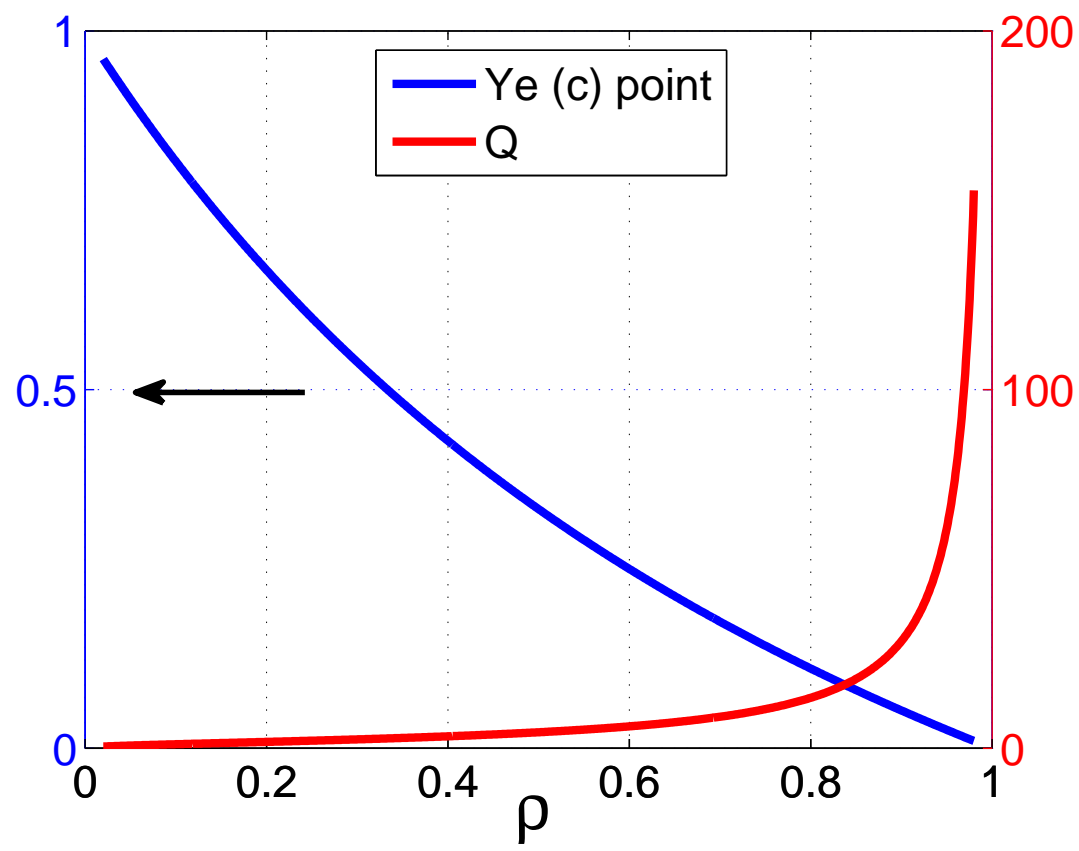

Fig. 8 Evolution of microring quality factor Q (red solid line) and reduced power level $Y_{e}$ (blue solid line) enabling mode conversion, as a function of the coupling parameter $\rho$. The parameters of the system are the same as in Fig. 6.

and nonlinear Kerr regimes. The presence of the microring breaks the intrinsic even/odd symmetry of the two-mode waveguide, thus enabling mode mixing. In the loss-less case, energy can be totally transferred from even to odd modes and vice versa, provided to operate on a resonance wavelength of the microring. The system can therefore act as a mode converter. We have also derived the analytical expression for the internal field of the microring and, when Kerr nonlinearity is effective, we have demonstrated mode-switching associated with ring phase bistability. One key hypothesis of our model relies on the existence of a phase matching condition between the microring propagation constant $\beta$ and the average value $\left(\beta_{\text {even }}+\beta_{\text {odd }}\right) / 2$ of the propagation constants of the even and odd modes in the two-mode waveguide. This condition gives rise to a particularly simple and compact form for the ternary coupler scattering matrix. When relaxing this constraint on $\beta$, a scattering matrix representation for the ternary coupler can be still derived analytically, but the detailed calculation proves to be too cumbersome to be presented in the frame of this work. Nevertheless, the main symmetries of the matrix are preserved, so that the main conclusions remain essentially the same, except for a slight shift of the resonant frequencies of the system. 


\section{A Considerations on the choice of the modal basis}

In Section 4, the matrix expression for the coupler has been obtained by treating the two-mode waveguide (TMWG) as a system of mutually coupled single-mode waveguides (SMWG), thus obtaining a description of the field evolutions in the fully single-mode basis $\left\{e_{1}, e_{2}, e_{3}\right\}$, where $e_{n}$ denotes the unit (normalized) vector associated to the mode propagating in the $n$-th single-mode channel. A possible alternative basis is the one using $\left\{e_{1}, e_{\text {even }}, e_{\text {odd }}\right\}$, in which $e_{\text {even }}$ (resp. $e_{\text {odd }}$ ) denotes the even (resp. odd) amplitudes of the modes in the two-mode waveguide (see Fig. 3). Within this alternative hybrid single/multimode framework, the evolution equation takes the following form:

$$
i \frac{\partial}{\partial z}\left(\begin{array}{c}
F_{1} \\
F_{\text {even }} \\
F_{\text {odd }}
\end{array}\right)=\left(\begin{array}{ccc}
\beta & \chi_{1 e} & \chi_{1 o} \\
\chi_{1 e} & \beta_{\text {even }} & 0 \\
\chi_{1 o} & 0 & \beta_{\text {odd }}
\end{array}\right)\left(\begin{array}{c}
F_{1} \\
F_{\text {even }} \\
F_{\text {odd }}
\end{array}\right)
$$

where $\beta$ denotes, as previously, the propagation constant of the upper SMWG, and $\beta_{\text {even }}$ (resp. $\beta_{\text {odd }}$ ) stands for the propagation constant of the even (resp. odd) mode in the lower TMWG. As far as coupling constants are concerned, we need an expression for both $\chi_{1 e}$ (between mode 1 and mode even) and $\chi_{1 o}$ (between mode 1 and mode odd). This means two degrees of freedom for a complete description of the coupler. Besides, thanks to their intrinsic orthogonality, no direct coupling between the even and odd modes needs to be accounted for.

On the other hand, within the framework exploited in Section (4), the TMWG is treated as a system of two mutually coupled SMWG interacting with the upper SMWG, the coupling being always limited to the nearest neighbour(s). For the sake of algebraic simplicity, the three SMWG are assumed identical, sharing therefore the same propagation constant $\beta$. The upper (resp. lower) coupling constant is denoted $\chi_{a}$ (resp. $\chi_{b}$ ). In this basis, according to Eq. (5):

$$
i \frac{\partial}{\partial z}\left(\begin{array}{l}
F_{1} \\
F_{2} \\
F_{3}
\end{array}\right)=\left(\begin{array}{ccc}
\beta & \chi_{a} & 0 \\
\chi_{a} & \beta & \chi_{b} \\
0 & \chi_{b} & \beta
\end{array}\right)\left(\begin{array}{l}
F_{1} \\
F_{2} \\
F_{3}
\end{array}\right) .
$$

The complex amplitudes of the modes can be transformed from one basis into the other by means of the following rotation matrices:

$$
\left(\begin{array}{c}
F_{1} \\
F_{\text {even }} \\
F_{\text {odd }}
\end{array}\right)=\left(\begin{array}{ccc}
1 & 0 & 0 \\
0 & \sqrt{2} / 2 & \sqrt{2} / 2 \\
0 & -\sqrt{2} / 2 & \sqrt{2} / 2
\end{array}\right)\left(\begin{array}{c}
F_{1} \\
F_{2} \\
F_{3}
\end{array}\right), \quad\left(\begin{array}{c}
F_{1} \\
F_{2} \\
F_{3}
\end{array}\right)=\left(\begin{array}{ccc}
1 & 0 & 0 \\
0 & \sqrt{2} / 2 & -\sqrt{2} / 2 \\
0 & \sqrt{2} / 2 & \sqrt{2} / 2
\end{array}\right)\left(\begin{array}{c}
F_{1} \\
F_{\text {even }} \\
F_{\text {odd }}
\end{array}\right) .
$$

As a result, we get eventually:

$$
i \frac{\partial}{\partial z}\left(\begin{array}{c}
F_{1} \\
F_{\text {even }} \\
F_{\text {odd }}
\end{array}\right)=\left(\begin{array}{ccc}
\beta & \chi_{a} / \sqrt{2} & -\chi_{a} / \sqrt{2} \\
\chi_{a} / \sqrt{2} & \beta+\chi_{b} & 0 \\
-\chi_{a} / \sqrt{2} & 0 & \beta-\chi_{b}
\end{array}\right)\left(\begin{array}{c}
F_{1} \\
F_{\text {even }} \\
F_{\text {odd }}
\end{array}\right) .
$$

Eq. (32) should be now compared with Eq. (30). Note that the three modes $\left\{e_{1}, e_{\text {even }}, e_{\text {odd }}\right\}$ do not propagate at the same velocity, and that the coupling between $e_{1}$ and $e_{\text {even }}$ has the same magnitude (but not the same sign) as that between $e_{1}$ and $e_{o d d}$. Not only do we recover the (rather obvious) fact that $\beta_{\text {even }}=\beta+\chi_{b}$ and $\beta_{\text {even }}=\beta-\chi_{b}$ : by identification, we also determine a compact expression for the coupling constants $\chi_{1 e}=-\chi_{1 o}=\chi_{a} / \sqrt{2}$. In other words, the approach dealing with single mode waveguides is simpler, as one less degree of freedom is required. Moreover, a multimode propagation problem looks more easily tractable and understandable in terms of coupled single-mode channels, thus giving an alternative picture with an immediate physical insight.

\section{B Intersection of a Lorentzian function with a straight line}

If the microring resonances are sharp enough to be assimilated to Lorentzian functions, then, with a suitable variable substitution, Eq. (26) can be recast into the following equivalent 
form:

$$
\frac{1}{x^{2}+1}=\frac{x+a}{K} \Leftrightarrow\left(x^{2}+1\right)(x+a)=K,
$$

modelling the intersection of a Lorentzian curve with a straight line of horizontal intercept (anchor point) $a$ and its inverse slope $K$, as depicted in Fig. 9.

Eq. (33) is polynomial equation of the third-order, which is algebraically solvable by means of the Cardano's formula, and whose roots are parametrized by $a$ and $K$. By operating the substitutions: $b=(a / 3), z=x+b, p^{\prime}=-b^{2}+(1 / 3)$ and $q^{\prime}=b^{3}+b-(K / 2)$, Eq. (33) can be recast into its reduced cubic form:

$$
z^{3}+3 p^{\prime} z+2 q^{\prime}=0
$$

which allows to investigate the nature of the roots more easily. More specifically, Eq. (33) admits three real (physical) solutions only if the factor:

$$
R=\left(p^{\prime}\right)^{3}+\left(q^{\prime}\right)^{2}=\left(K^{\prime}\right)^{2}-2 K^{\prime} b\left(b^{2}+1\right)+\frac{18 b^{2}+1}{27}, \quad K^{\prime}=K / 2
$$

is strictly negative $(R<0)$

The condition on the negative sign for $R$ is fulfilled only if $p^{\prime}<0$, that is if $a>\sqrt{3}$. Therefore, in order to have $R<0$ and thus three real solutions, the anchor point $a$ of the straight line must be located well outside the Lorentzian line shape. This first condition on $a$ being assured, the factor $R$ is negative for values of the reduced slope $K^{\prime}$ lying within the two roots of the polynomial (35). The explicit values for these roots can be evaluated through the reduced discriminant:

$$
\Delta^{\prime}=b^{2}\left(b^{2}+1\right)-\frac{18 b^{2}+1}{27}
$$

which is a quantity unconditionally positive since $b>(1 / 3)$. The two roots $K_{1,2}^{\prime}$ of (35) are finally: $K_{1,2}^{\prime}=b\left(b^{2}+1\right) \pm \sqrt{\Delta^{\prime}}$.

To summarize, for a given detuning $a>\sqrt{(3)}$, the bistability range of the system described by Eq. (33) corresponds to the zone where the reduced slope $K^{\prime}$ lies between the two roots $K_{1,2}$ of (35), where $R<0$.

Acknowledgements This work is supported by the Labex CominLabs (ANR-10-LABX07-01) through the 3D-Optical-ManyCores project: (http://www.3d-opt-many-cores.cominlabs.ueb.eu/). The authors would like to thank Prof. Christophe Peucheret for helpful discussions.

\section{References}

1. Bergman, K., Carloni, L.P., Biberman, A., Chan, J., Hendry, G.: Photonic Network-onChip Design. Springer-Verlag New York (2014). DOI 10.1007/978-1-4419-9335-9

2. Boucher, Y.G.: Analytical model for the coupling constant of a directional coupler in terms of slab waveguides. Optical Engineering 53(7), 071,810 (2014). DOI 10.1117/1.OE.53.7.071810. URL http://dx.doi.org/10.1117/1.OE.53.7.071810

3. Boyd, R.W.: Nonlinear Optics, 3 edn. Academic Press (2008)

4. Chen, S., Zhang, L., Fei, Y., Cao, T.: Bistability and self-pulsation phenomena in silicon microring resonators based on nonlinear optical effects. Opt. Express 20(7), 7454-7468 (2012). DOI 10.1364/OE.20.007454. URL http://www.opticsexpress.org/abstract.cfm?URI=oe-20-7-7454

5. Ding, Y., Xu, J., Ros, F.D., Huang, B., Ou, H., Peucheret, C.: On-chip two-mode division multiplexing using tapered directional coupler-based mode multiplexer and demultiplexer. Opt. Express 21(8), 10,376-10,382 (2013). DOI 10.1364/OE.21.010376. URL http://www.opticsexpress.org/abstract.cfm?URI=oe-21-8-10376 


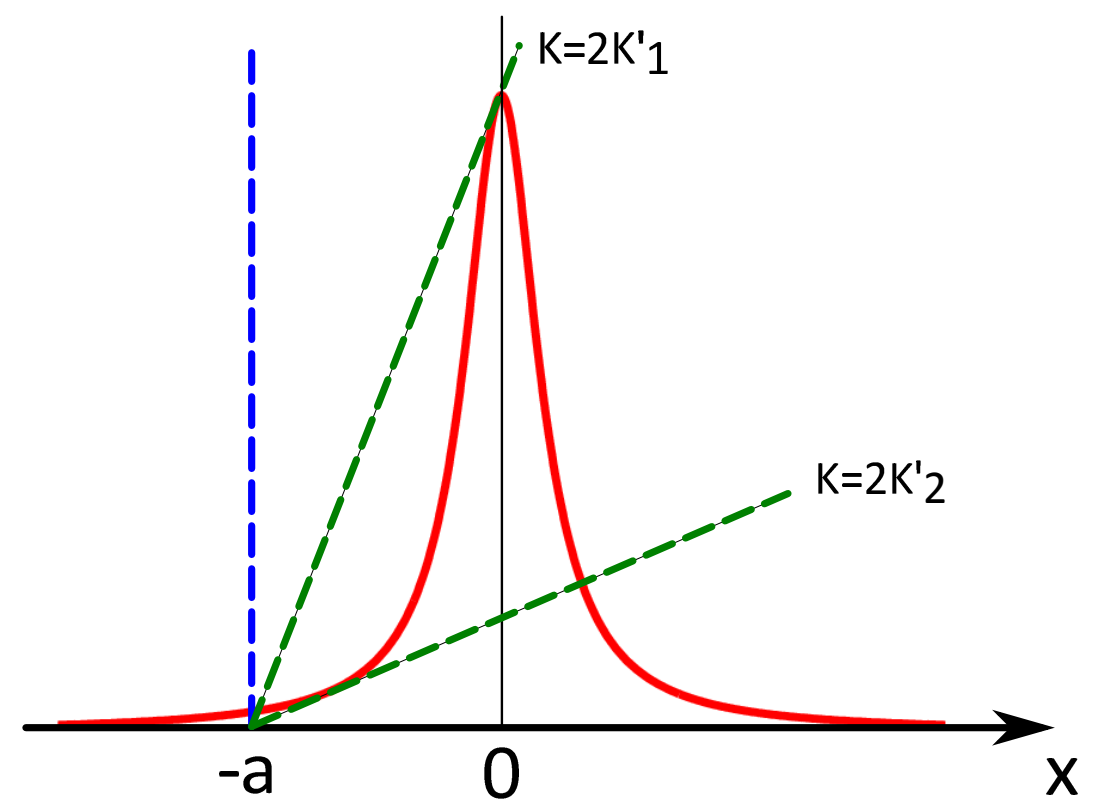

Fig. 9 Intersection of a straight line of variable slope $K$ and anchor point $a$ with a Lorentzian curve. The vertical blue dashed line correspond to the linear regime, whereas the two green-dashed lines delimit the range of bistability inside which two or three distinct real solutions may exist.

6. Dumeige, Y., Féron, P.: Dispersive tristability in microring resonators. Phys. Rev. E 72, 066,609 (2005). DOI 10.1103/PhysRevE.72.066609. URL http://link.aps.org/doi/10.1103/PhysRevE.72.066609

7. Luo, L.W., Ophir, N., C.P.Chen, Gabrielli, L., Poitras, C., Bergman, K., Lipson, M.: WDM-compatible mode-division multiplexing on a silicon chip. Nature Communications 5(3069) (2014). DOI 10.1038/ncomms4069

8. Matsko, A.B., Savchenkov, A.A., Strekalov, D., Ilchenko, V.S., Maleki, L.: Optical hyperparametric oscillations in a whispering-gallery-mode resonator: Threshold and phase diffusion. Phys. Rev. A 71, 033,804 (2005). DOI 10.1103/PhysRevA.71.033804. URL http://link.aps.org/doi/10.1103/PhysRevA.71.033804

9. Miller, D.A.B.: Device Requirements for Optical Interconnects to Silicon Chips. Proceedings of the IEEE 97(7), 1166-1185 (2009). DOI 10.1109/JPROC.2009.2014298

10. Parini, A., Bellanca, G., Annoni, A., Morichetti, F., Melloni, A., Strain, M., Sorel, M., Gay, M., Pareige, C., Bramerie, L., Thual, M.: BER evaluation of a passive SOI WDM router. Photonics Technology Letters, IEEE 25(23), 2285-2288 (2013). DOI 10.1109/LPT.2013.2285248

11. Peucheret, C., Ding, Y., Xu, J., Ros, F.D., Parini, A., Ou, H.: Signal processing for On-Chip Space Division Multiplexing. In: Advanced Photonics 2015, p. SpT2E.3. Optical Society of America (2015). DOI 10.1364/SPPCOM.2015.SpT2E.3. URL http://www.osapublishing.org/abstract.cfm?URI=SPPCom-2015-SpT2E.3

12. Poon, A., Luo, X., Xu, F., Chen, H.: Cascaded Microresonator-Based Matrix Switch for Silicon On-Chip Optical Interconnection. Proceedings of the IEEE 97(7), 1216-1238 (2009). DOI 10.1109/JPROC.2009.2014884

13. Rasoloniaina, A., Huet, V., Thual, M., Balac, S., Féron, P., Dumeige, Y.: Analysis of third-order nonlinearity effects in very high-q wgm resonator cavity ringdown spec- 
troscopy. J. Opt. Soc. Am. B 32(3), 370-378 (2015). DOI 10.1364/JOSAB.32.000370. URL http://josab.osa.org/abstract.cfm?URI=josab-32-3-370

14. Rukhlenko, I.D., Premaratne, M., Agrawal, G.P.: Analytical study of optical bistability in silicon ring resonators. Opt. Lett. 35(1), 55-57 (2010). DOI 10.1364/OL.35.000055. URL http://ol.osa.org/abstract.cfm?URI=ol-35-1-55

15. Sarid, D.: Analysis of bistability in a ring-channel waveguide. Opt. Lett. 6(11), 552-553 (1981). DOI 10.1364/OL.6.000552. URL http://ol.osa.org/abstract.cfm?URI=ol-6-11552

16. Shacham, A., Bergman, K., Carloni, L.: Photonic Networks-on-Chip for Future Generations of Chip Multiprocessors. Computers, IEEE Transactions on 57(9), 1246-1260 (2008). DOI 10.1109/TC.2008.78

17. Sherwood-Droz, N., Wang, H., Chen, L., Lee, B.G., Biberman, A., Bergman, K., Lipson, M.: Optical $4 \times 4$ hitless silicon router for optical Networks-on-Chip (NoC). Opt. Express 16(20), 15,915-15,922 (2008). DOI 10.1364/OE.16.015915. URL http://www.opticsexpress.org/abstract.cfm?URI=oe-16-20-15915

18. Stern, B., Zhu, X., Chen, C.P., Tzuang, L.D., Cardenas, J., Bergman, K., Lipson, M.: On-chip mode-division multiplexing switch. Optica 2(6), $\quad 530-535 \quad$ (2015). $\quad$ DOI 10.1364/OPTICA.2.000530. URL http://www.osapublishing.org/optica/abstract.cfm?URI=optica-2-6-530

19. Sun, C., Yu, Y., Chen, G., Zhang, X.: Integrated switchable mode exchange for reconfigurable mode-multiplexing optical networks. Opt. Lett. 41(14), 3257-3260 (2016). DOI 10.1364/OL.41.003257. URL http://ol.osa.org/abstract.cfm?URI=ol-41-14-3257

20. Tamir, T.: Guided-Wave Optoelectronics. Springer-Verlag New York (1988)

21. Treussart, F., Ilchenko, V., Roch, J.F., Hare, J., Lefèvre-Seguin, V., Raimond, J.M., Haroche, S.: Evidence for intrinsic kerr bistability of high-q microsphere resonators in superfluid helium. The European Physical Journal D - Atomic, Molecular, Optical and Plasma Physics 1(3), 235-238 (1998). DOI 10.1007/PL00021556. URL http://dx.doi.org/10.1007/PL00021556

22. Yang, L., Xia, Y., Zhang, F., Chen, Q., Ding, J., Zhou, P., Zhang, L.: Reconfigurable nonblocking 4-port silicon thermo-optic optical router based on Mach-Zehnder optical switches. Opt. Lett. 40(7), 1402-1405 (2015). DOI 10.1364/OL.40.001402. URL http://ol.osa.org/abstract.cfm?URI=ol-40-7-1402

23. Yang, M., Green, W.M.J., Assefa, S., Campenhout, J.V., Lee, B.G., Jahnes, C.V., Doany, F.E., Schow, C.L., Kash, J.A., Vlasov, Y.A.: Non-Blocking 4x4 Electro-Optic Silicon Switch for On-Chip Photonic Networks. Opt. Express 19(1), 47-54 (2011). DOI 10.1364/OE.19.000047. URL http://www.opticsexpress.org/abstract.cfm?URI=oe-19$1-47$

24. Yang, Y.D., Li, Y., Huang, Y.Z., Poon, A.W.: Silicon nitride three-mode division multiplexing and wavelength-division multiplexing using asymmetrical directional couplers and microring resonators. Opt. Express 22(18), 22,172-22,183 (2014). DOI 10.1364/OE.22.022172. URL http://www.opticsexpress.org/abstract.cfm?URI=oe-22$18-22172$

25. Yariv, A., Yeh, P.: Optical Waves in Crystals. Wiley (1984)

26. Ye, M., Yu, Y., Sun, C., Zhang, X.: On-chip data exchange for mode division multiplexed signals. Opt. Express 24(1), 528-535 (2016). DOI 10.1364/OE.24.000528. URL http://www.opticsexpress.org/abstract.cfm?URI=oe-24-1-528

27. Zhang, C., Zhang, S., Peters, J.D., Bowers, J.E.: $8 \times 8 \times 40$ gbps fully integrated silicon photonic network on chip. Optica 3(7), $\quad 785-786 \quad$ (2016). $\quad$ DOI 10.1364/OPTICA.3.000785. URL http://www.osapublishing.org/optica/abstract.cfm?URI=optica-3-7-785 